\title{
Clinical follow-up on a cohort of patients with deficiency of adenosine deaminase 2 (DADA2)
}

\author{
K Barron $^{1 *}$, A Ombrello², D Stone ${ }^{2}$, P Hoffmann², I Aksentijevich², Q Zhou², A Jones², D Kastner ${ }^{2}$ \\ From 8th International Congress of Familial Mediterranean Fever and Systemic Autoinflammatory Diseases \\ Dresden, Germany. 30 September - 3 October 2015
}

\section{Introduction}

We previously reported a syndrome of intermittent fevers, early-onset lacunar strokes, livedoid rash, hepatosplenomegaly, immune deficiency, and systemic vasculopathy, associated with loss-of-function mutations in CECR1. An additional report by others expanded the clinical spectrum to include patients with cutaneous polyarteritis nodosa.

\section{Objectives}

We now present clinical follow-up on our reported 5 patients and an additional 9 patients.

\section{Patients and methods}

We evaluated the 14 patients at the National Institutes of Health. All patients were enrolled in an IRB approved study. We performed whole-exome sequencing in the initial 3 patients and their unaffected parents and candidate-gene sequencing in the other 11 patients. Clinical information and radiographic and laboratory testing were obtained at each visit.

\section{Results}

All patients had 2 mutations in CECR1. 11/14 patients reported recurrent fevers.

$10 / 14$ patients had at least one stroke, with $8 / 10$ before the age of 5 years. Magnetic resonance imaging showed evidence of acute or chronic small subcortical infarcts involving the deep-brain nuclei and the brain stem, consistent with smallvessel occlusions (lacunar strokes). Three patients had additional hemorrhagic strokes. In 10/10 patients, magnetic angiography showed no evidence of cerebral vasculitis.

All 14 patients demonstrated livedo racemosa. Erythematous papules or nodules were seen in 11 of these patients.

Hepato- and/or splenomegaly was observed in 10/14 with 3 patients demonstrating portal hypertension. One patient developed a perforated small bowel requiring resection.

Hypertension was noted in 2 patients. Prolonged QT was reported in 3 patients.

12/14 demonstrated hematologic abnormalities including anemia, leukopenia, and/or thrombocytopenia. Elevation of acute phase reactants was reported in 13/14 patients.

Low serum iron was noted in $8 / 10$ patients tested.

$10 / 13$ presented with hypogammaglobulinemia, however, this may reflect prior treatment with cyclophosphamide in 3 patients.

Most patients had received a number of medications over the course of their disease. It was our practice to discontinue aspirin and/or anticoagulation in all of our DADA2 patients. We observed striking improvement in CRP, ESR, CBC, and serum iron in $10 / 12$ patients receiving anti-TNF agents.

\section{Conclusion}

We have expanded the clinical picture of our cohort of patients with DADA2 to include multiple strokes, livedo racemosa, cutaneous PAN, portal hypertension, hematologic abnormalities, vascular pathology and mild immunodeficiency. In addition, we have demonstrated both clinical and laboratory improvement following treatment with anti-TNF agents.

\section{Authors' details}

${ }^{1} \mathrm{NIAID}, \mathrm{NIH}$, Bethesda, USA. ${ }^{2} \mathrm{NHGRI}, \mathrm{NIH}$, Bethesda, USA.

Published: 28 September 2015

doi:10.1186/1546-0096-13-S1-O21

Cite this article as: Barron et al.: Clinical follow-up on a cohort of patients with deficiency of adenosine deaminase 2 (DADA2). Pediatric Rheumatology 2015 13(Suppl 1):021. 